\title{
Polaron framework to account for transport properties in metallic epitaxial manganite films
}

\author{
P. Graziosi,,${ }^{1,2, *}$ A. Gambardella, ${ }^{1}$ M. Prezioso, ${ }^{1}$ A. Riminucci, ${ }^{1}$ I. Bergenti, ${ }^{1}$ N. Homonnay, ${ }^{3}$ G. Schmidt,${ }^{3,4}$ \\ D. Pullini, ${ }^{5}$ and D. Busquets-Mataix ${ }^{2,6}$ \\ ${ }^{1}$ CNR-ISMN, Consiglio Nazionale delle Ricerche-Istituto per lo Studio dei Materiali Nanostrutturati, v. Gobetti 101, 40129, Bologna, Italy \\ ${ }^{2}$ Instituto de Tecnología de Materiales, Universitat Politécnica de Valencia, Camino de Vera s/n, 46022, Valencia, Spain \\ ${ }^{3}$ Institut für Physik, Universität Halle, 06120 Halle, Germany \\ ${ }^{4}$ Center for Materials Science, Universität Halle, 06120 Halle, Germany \\ ${ }^{5}$ Centro Ricerche Fiat, 10043, Orbassano, Torino, Italy \\ ${ }^{6}$ Departamento de Ingeniería Mecánica y de Materiales, Universitat Politécnica de Valencia, Camino de Vera s/n, 46022, Valencia, Spain
}

(Received 17 March 2014; revised manuscript received 30 April 2014; published 12 June 2014)

\begin{abstract}
We propose a model for the consistent interpretation of the transport behavior of manganese perovskites in both the metallic and insulating regimes. The concept of polarons as charge carriers in the metallic ferromagnetic phase of manganites also solves the conflict between transport models, which usually neglects polaron effects in the metallic phase, and, on the other hand, optical conductivity, angle-resolved spectroscopy, and neutron scattering measurements, which identify polarons in the metallic phase of manganites down to $6 \mathrm{~K}$. Transport characterizations of epitaxial $\mathrm{La}_{0.7} \mathrm{Sr}_{0.3} \mathrm{MnO}_{3}$ thin films in the thickness range of 5-40 nm and temperature interval of $25-410 \mathrm{~K}$ have been accurately collected. We show that taking into account polaron effects allows us to achieve an excellent fit of the transport curves in the whole temperature range. The current carriers density collapse picture accurately accounts for the properties variation across the metal-insulator transitions. The electron-phonon coupling parameter $\gamma$ estimations are in a good agreement with theoretical predictions. The results promote a clear and straightforward quantitative description of the manganite films involved in charge transport device applications and promises to describe other oxide systems involving a metal-insulator transition.
\end{abstract}

DOI: 10.1103/PhysRevB.89.214411 PACS number(s): 75.47.Lx, 75.47.Gk, 73.50.-h, 71.38.-k

Ferromagnetic manganites are a prototypical example of the so-called half-metallic materials, i.e., materials with $100 \%$ spin polarization at $0 \mathrm{~K}$. Although their possible application in commercial spintronics is prevented by a relatively low Curie temperature $\left(T_{C} \leqslant 370 \mathrm{~K}\right)$, manganites represent an ideal laboratory tool to test spin transport in various materials and to search for pioneering device paradigms [1,2]. Thus, the use of manganites have significantly contributed to the field of organic spintronics, where almost half of the reported devices have $\mathrm{La}_{0.7} \mathrm{Sr}_{0.3} \mathrm{MnO}_{3}$ (LSMO) as an injector [3]. In this context, revealing in a most exhaustive and comprehensive way, the transport properties of these materials looks both captivating and thought provoking.

The transport properties of manganites are strongly linked to their ferromagnetism and are generally described in the framework of the so-called double-exchange mechanism: below $T_{C}$ the exchange interaction between $\mathrm{Mn}$ cations through oxygen anions favors ferromagnetism and electron delocalization along the Mn-O-Mn bonds, while above $T_{C}$, thermal disorder disrupts this delocalization leading to a metal-insulator transition (MIT) [4]. Nevertheless, this basic picture appears to be conceptually insufficient to describe the physics of manganites, in general, and LSMO, in particular [5]: the strong role of polaron effects in manganites has been pointed out [6], and good experimental evidence has been provided by a variety of methods [6-13]. Moreover, manganites were proposed as an example of a polaron Fermi liquid [7].

Although the presence of polarons in the metallic phase of LSMO (down to $6 \mathrm{~K}$ ) has been demonstrated by optical

\footnotetext{
*patrizio.graziosi@gmail.com
}

conductivity and reflectivity $[8,9]$ and a polaron metal phase has been indicated in metallic $\mathrm{La}_{2-2 x} \mathrm{Sr}_{1+2 x} \mathrm{Mn}_{2} \mathrm{O}_{7}$ by angleresolved photoemission spectroscopy [14] and neutron scattering [15], explicit evidence of the polaron effects from transport characterizations was, up to now, still missing. Indeed, despite these convincing proves, the dominating trend in literature is to describe the resistivity of manganites via a $T^{2}+T^{5}$ behavior, ignoring, thus, the polaron effects (see, for instance, a very recent communication in Ref. [16]). The few attempts to include polarons in the transport description of the manganites were mainly oversimplified, leaving open the issue of real nature of charge carriers $[17,18]$.

The standard polaron theory states that at low temperature, polarons can form a band and move without thermal activation, carrying their polarization with them [19]. The polaron band narrows with increasing temperature due to the corresponding increase of the polaron effective mass. When the bandwidth is comparable with $k_{B} T$, the band picture breaks down, and the transport becomes thermally activated. This happens at the transition temperature $T_{t} \approx \frac{\hbar \omega_{0}}{2 k_{B}}$, where $\hbar \omega_{0}$ is the dominant phonon mode coupled to the electron [20]. The polaron band narrowing due to the increase of the polaron effective mass in ionic materials is a known effect and is described by the Sewell model by taking into account the atomicity of the lattice [20,21].

As mentioned above, most of the attempts tend to promote the description of the transport in LSMO without involving the polaron effects, though including the electron phonon interaction in the transport equations in four different ways.

(i) A simple combination of electron-phonon interaction $T^{5}$ term and electron-electron interaction $T^{2}$ term in $R(T)=$ $R_{0}+R_{2} T^{2}+R_{5} T^{5}$ is often performed neglecting that the $T^{5}$ dependence of the electron-phonon interaction is just 
a simplification of the case of a monovalent metal with a spherical Fermi surface and an oversimplified representation of the electron-phonon interaction matrix [22]; indeed the $T^{5}$ resistivity coefficient appears to vary considerably with temperature and is determined by imposing the $T^{5}$ dependence in limited temperature ranges, even in alkali metals [22]. This description of the electron phonon coupling appears even more inappropriate for LSMO, which has different valence states, carrier concentration, and nonspherical Fermi surface [23]. When applied [24-28], this approach gave unrealistic values for the $T^{5}$ coefficient, which is found to be one to two orders of magnitude lower than in metals $[29,30]$. These findings are in obvious contradiction with the strong electronphonon coupling, known to govern the physics of manganites $[6,12,18,31-35]$. The basic problem is nevertheless the clear failure of this approach to fit the main $R(T)$ features when applied in an extended temperature range, as represented in Fig. 3.

(ii) In the second approach, the electron-magnon interaction $T^{9 / 2}$ term [36] substitutes the $T^{5}$ in the $T^{2}+T^{5}$ approach. The treatment of the electron-magnon scattering that leads to the $T^{9 / 2}$ term was conducted by neglecting explicitly the coupling between electrons and phonons and holding the ions rigidly in their equilibrium position [36]; thus, it appears to not be suitable for manganites, whose physics is governed by electron-phonon coupling. Indeed, when applied, this does not improve the accuracy of the fit, requiring $T^{9 / 2}$ coefficients too low to be physically meaningful $[28,37]$.

(iii) Even the use of a more generic $T^{\alpha}$ term, where $\alpha$ is a fitting parameter without a clear link to a scattering mechanism, does not lead to significant improvements [38].

(iv) Finally, an accurate use of the Bloch-Grüneisen integral was attempted by Varshney et al. [17,18,34,35,39-42]. Their model was found to fit very well the experimental data measured on polycrystalline samples [17,18,34,39-43], while for epitaxial films, a good fit could be achieved only at low temperatures [35].

As mentioned above, the attempts at inserting polaron effects in the transport equations were somehow simplistic. Thus, in one of the very few available communications, the polarons have been included by considering the general expression for the mobility $\mu=\frac{e \tau}{m^{*}}$, where $e, m^{*}$, and $\tau$ are, respectively, the electronic charge, the effective mass, and the scattering time, and $\tau$ is assumed to correspond to a polaron behavior $[44,45]$. A successful fit was achieved only below $100 \mathrm{~K}$, although the authors used the expression for the scattering time derived by Lang and Firsov [46] for insulating polarons instead of that for the metallic case [47]. Even the application of the model for this temperature interval was not clearly justified. Indeed, the fit defined the value $\frac{\hbar \omega_{0}}{k_{B}} \approx 80 \mathrm{~K}$, and given that the applicability condition of the model is $T \ll \frac{\hbar \omega_{0}}{k_{B}}$, the analysis should have to be limited to much lower temperatures.

Thus, the clear contradiction between transport and optical data sheds doubts on the real role of polarons in these materials and requires an accurate investigation. We show in this paper that transport in high-quality LSMO thin films is fully compatible with the polaron picture, when properly constructed, which provides an excellent fit with physically meaningful and realistic parameters. (a)

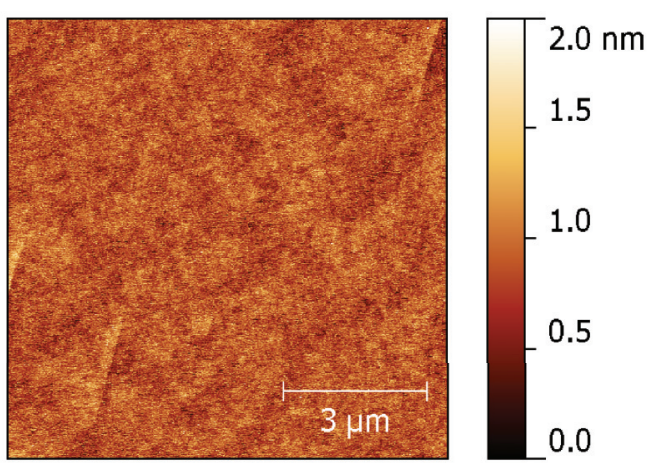

(b)

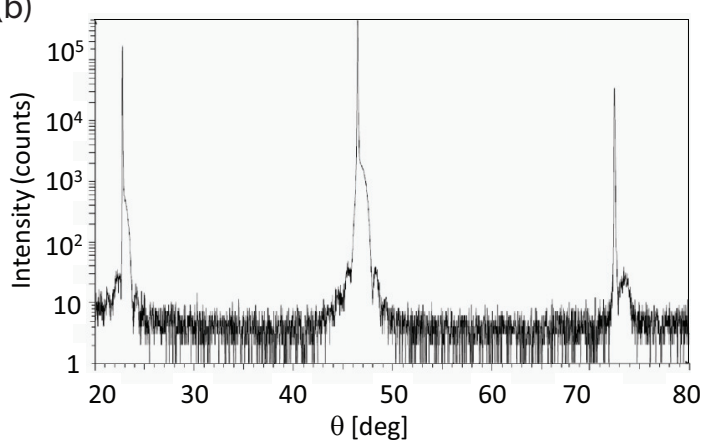

FIG. 1. (Color online) Characterization of a 12-nm-thick LSMO deposited on STO (100): (a) $9 \times 9 \mu \mathrm{m}^{2}$ AFM image and (b) XRD pattern showing (001), (002), and (003) reflections.

LSMO epitaxial thin films were deposited by channel-spark ablation on $\mathrm{SrTiO}_{3}$ (100) (STO) and $\mathrm{NdGaO}_{3}$ (110) (NGO) substrates [48]. The $R(T)$ measurements were performed in a closed-cycle helium cryostat with four contacts made by silver paste [45]. The polarity inversion method has been used in order to rule out systematic errors [49]. All the measurements start at $25 \mathrm{~K}$, and the heating rate is between 0.2 and $0.3 \mathrm{~K} / \mathrm{min}$. The electronic structure of the films used in this work, briefly shown elsewhere [50], is very similar to that known for single crystals, demonstrating the high quality of the films and, as a consequence, the general validity of the proposed model.

Figure 1(a) shows a $9 \times 9 \mu \mathrm{m}^{2}$ atomic force microscopy (AFM) image of the LSMO surface for a typical $12 \mathrm{~nm}$ film deposited on STO substrate: the peak to valley corrugation is confined $2 \mathrm{~nm}$, and the root mean square (rms) roughness is $0.25 \mathrm{~nm}$. On a larger area, some particulate originating from the ablation process increases the overall rms roughness to $0.6-0.7 \mathrm{~nm}$. The epitaxial quality of the samples is shown by the x-ray diffraction (XRD) pattern: Fig. 1(b) shows the data for a typical $12 \mathrm{~nm}$ LSMO film grown on STO. The (001), (002), and (003) reflections are detected. The peaks of the film are superimposed with the ones of the substrate, demonstrating the epitaxial quality. Figure 2 shows the resistivity data of some films versus $T / T_{M I}$; this choice is motivated by the MIT temperature being perceived as the most relevant parameters for the electronic properties of manganites, regardless they are characterized by intermediate or weak electron-phonon coupling [51-53]. The resistivity values are perfectly in line with the ones known for LSMO films [54,55]. Figures 3 to 5 show detailed experimental 


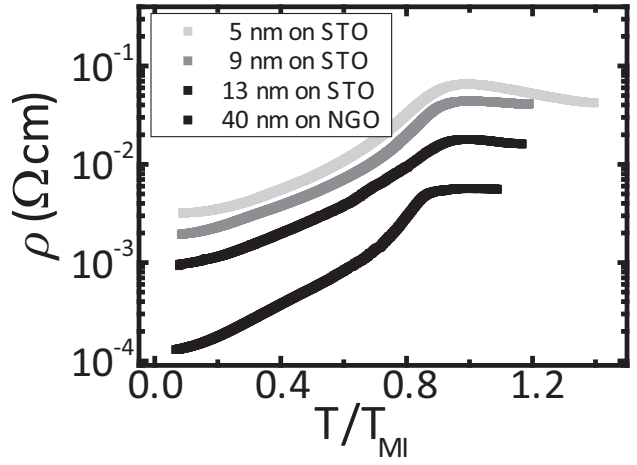

FIG. 2. Resistivity data of selected films plotted versus $T / T_{M I}$. The trend and the values are in line with the state of the art. The values of the $T_{M I}$ for each thickness value can be obtained from the individual graphs.

data about the resistance as a function of temperature (in log-log scale) collected in $0.1 \mathrm{~K}$ steps. While we note that the temperature trend is generally akin to most of the data available in the literature, we concentrate on an explicit quantitative treatment of these curves. We choose to fit the bare measured quantity, i.e., resistance instead of resistivity, since we aim to focus on the temperature behavior of the measured quantity and there is only a geometrical factor [56].

In order to demonstrate the validity of the polaron metal transport model proposed in this paper and to assert its general relevance, the model is successfully applied to the metallic phase of single crystals of $\mathrm{La}_{0.67} \mathrm{Ca}_{0.33} \mathrm{MnO}_{3}$ (LCMO), $\mathrm{La}_{0.67}\left(\mathrm{Ca}_{1 / 3} \mathrm{~Pb}_{2 / 3}\right)_{0.33} \mathrm{MnO}_{3}$ (LCPMO), and LSMO, taking the data from the literature [57], and the showing the results in Fig. 6. Moreover, the current carrier density collapse (CCDC) model has been applied to fit the data on LCMO in the whole temperature range.

We first analyze the metallic parts of the $R(T)$ curves and apply Matthiessen's rule to the transport properties. In this framework, the mobility $\mu$ is described as $\frac{1}{\mu}=\sum_{i} \frac{1}{\mu_{i}}$, where $i$ represents a specific scattering mechanism with its temperature

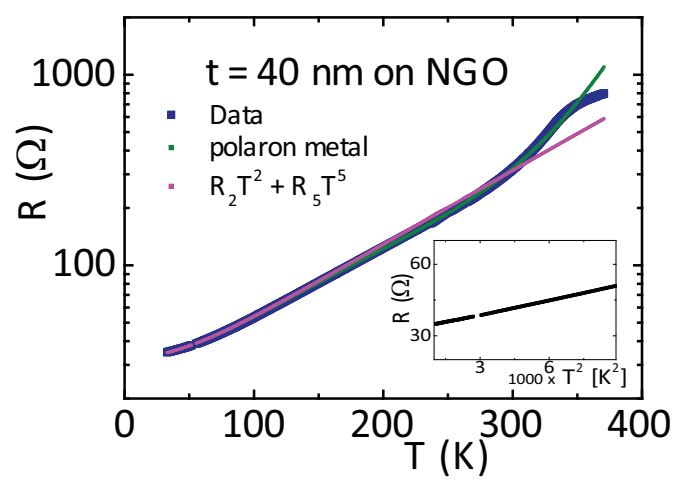

FIG. 3. (Color online) Transport data of a $40 \mathrm{~nm}$ LSMO/NGO film; the fits with the polaron and polynomial models are presented. While the polaron metal model [Eq. (2)] features an excellent agreement with the data, the polynomial model shows poor agreement and unrealistic values of the coefficients. The inset shows the linearization of the low $T$ data when plotted versus $T^{2}$ from which we obtain the $R_{0}$ and $R_{2}$ coefficients.

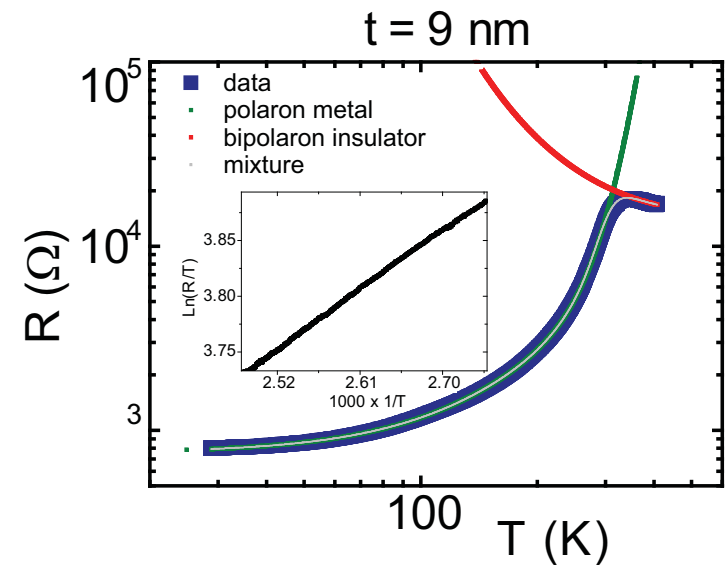

FIG. 4. (Color online) Transport data and fits for a $9 \mathrm{~nm} \mathrm{LSMO}$ and STO thin film; the metallic side is fitted with Eq. (2), while the insulating branch is described by thermal activated adiabatic hopping, which linearizes the data above the MIT (inset). The whole $R(T)$ is fitted by Eq. (3).

dependence [58]. Furthermore, we consider the electrical resistance to be proportional to the inverse of the mobility and assume $\mu=\frac{e \tau}{m^{*}}$, where $e, m^{*}$, and $\tau$ are, respectively, the electronic charge, the effective mass, and the scattering time.

First, we note that $R(T)$ data can be linearized by plotting $R$ versus $T^{2}$ in a relatively wide range of temperatures, approximately for $25<T<60-80 \mathrm{~K}$, depending on film thickness. It should be noted that such an unusually wide interval of validity for a pure $T^{2}$ dependence is quite characteristic for strongly correlated materials [59-63], although its origin is not yet fully understood [59]. In this paper, we take advantage of this to graphically define the coefficients $R_{0}$ and $R_{2}$.

In order to understand the transport above $80 \mathrm{~K}$, we introduce the electron-phonon interaction. We observe that in strongly polarizable materials, such as oxides, it is sufficient to consider only the contribution to the electronphonon interaction of optical phonons [64]. Assuming the Einstein model for lattice vibration, we limit the treatment at only one optical phonon $\hbar \omega_{0}$. Thus, the effective mass of the polaron exponentially increases with temperature as $m^{*}=m_{0} \exp \left[g^{2} \operatorname{coth}\left(\frac{\hbar \omega_{0}}{2 k_{B} T}\right)\right][20,21,65]$, where $m_{0}$ is the free electron mass and $g^{2}=\gamma \frac{E_{S P}}{\hbar \omega_{0}}$ is the zero temperature band-narrowing factor: polaron bandwidth narrows as $w=$ $w_{0} \exp \left[g^{2} \operatorname{coth}\left(\frac{\hbar \omega_{0}}{2 k_{B} T}\right)\right]$, where $w_{0}$ is the electron half bandwidth $[20,21,65] . E_{S P}$ is the polaron level shift and represents the amount by which the middle electronic band falls as a result of the potential well created by the lattice deformation, and $\gamma$ is the dimensionless electron-phonon interaction parameter. $E_{S P}$ in LSMO is estimated to be about $0.85 \mathrm{eV}$ [32] and $\gamma$ is expected to be less than unity [64]. In the fit, we set $\hbar \omega_{0}=$ $425 \mathrm{~cm}^{-1}$, which is the most characteristic phonon mode in metallic rhombohedral LSMO [11,66,67].

In addition to the effective mass approach, we adopt for the scattering time of the electron-phonon interaction an expression working in narrow bands, where two-phonon scattering is important, $\frac{1}{\tau_{e-p h}} \approx w \gamma^{4} n_{\omega}\left(n_{\omega}+1\right) ; w$ is the bandwidth, $\gamma$ the electron-phonon coupling parameter, and $n_{\omega}=1 /\left(\exp \left(\frac{\hbar \omega}{k_{B} T}\right)-1\right)$ [68]. The description of the lattice 

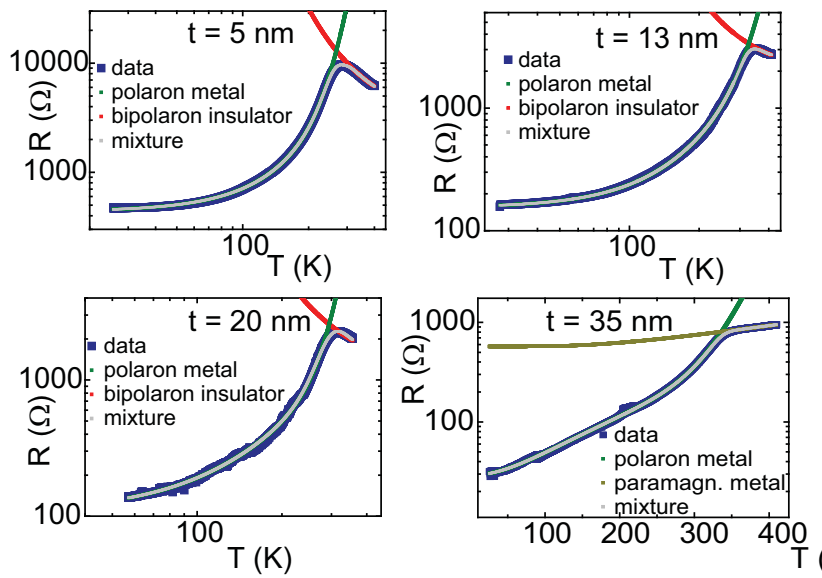

FIG. 5. (Color online) Transport data and fits of films of different thickness t deposited on STO. The metallic side is fitted with Eq. (2), while the insulating branch is described by thermal activated adiabatic hopping; the 35-nm-thick film seems to present a metal-to-metal transition, and the metallic data at the higher temperatures have been fitted using Eq. (2) but with a constant effective mass. The whole $R(T)$ is fitted by Eq. (3).

effect on the mobility becomes, thus,

$$
\begin{aligned}
\frac{1}{\mu_{e-p h}} & \approx m^{*} \frac{1}{\tau_{e-p h}} \approx m^{*} w \gamma^{4} n_{\omega}\left(n_{\omega}+1\right) \\
& \propto \exp \left[2 g^{2} \operatorname{coth}\left(\frac{\hbar \omega_{0}}{2 k_{B} T}\right)\right] \frac{\exp \left(\frac{\hbar \omega_{0}}{k_{B} T}\right)}{\left(\exp \left(\frac{\hbar \omega_{0}}{k_{B} T}\right)-1\right)^{2}} .
\end{aligned}
$$

Together with the $T^{2}$ dependence, empirically found, and considering Matthiessen's rule $\frac{1}{\mu}=\sum_{i} \frac{1}{\mu_{i}}, R \propto \frac{1}{\mu}, R=$ $\sum_{i} R_{i}$, one comes to the final expression for the metallic branch $R_{M}(T)$ of the total $R(T)$ :

$$
\begin{aligned}
R_{M}(T)= & R_{0}+R_{2} T^{2}+C_{F M} \exp \left[2 g^{2} \operatorname{coth}\left(\frac{\hbar \omega_{0}}{2 k_{B} T}\right)\right] \\
& \times \frac{\exp \left(\frac{\hbar \omega_{0}}{k_{B} T}\right)}{\left(\exp \left(\frac{\hbar \omega_{0}}{k_{B} T}\right)-1\right)^{2}} .
\end{aligned}
$$

The real fitting parameters are only $g^{2}$ and $C_{F M}$, the latter representing the proportionality constant between the resistance and the inverse of the electron-phonon dependent mobility ( $F M$ refers to the ferromagnetic phase); $R_{0}$ and $R_{2}$ are obtained graphically, and $\hbar \omega_{0}$ was set as a constant as stated above. We stress that we consider the carrier density to be constant; we show later that this approximation is consistent.

The fitting results of the $R_{M}(T)$ with this model are shown in Figs. 3-5 for six different films, and the agreement between the experimental data and the model is excellent (green lines). Very similar $\gamma$ values $(0.33-0.35)$ have been obtained for four samples out of the five deposited on STO, while it was slightly lower for the NGO case (0.27). Although, to the best of our knowledge, there are no other experimental measurements of the dimensionless $\gamma$ for manganites, these are reliable values for manganites because they are similar to the ones for cuprates, about 0.3 , as expected from theoretical consideration

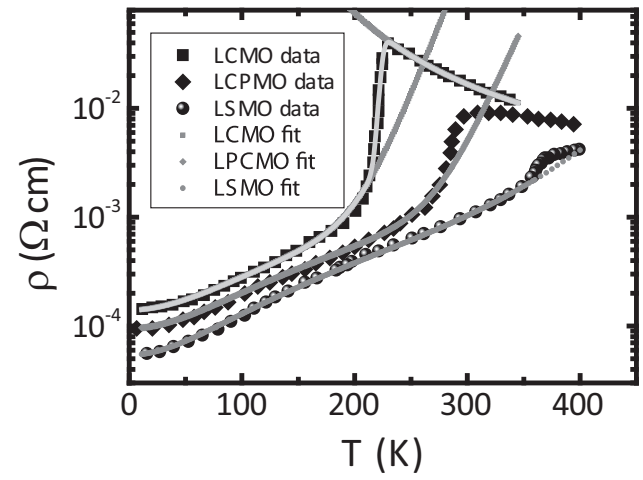

FIG. 6. Fits of transport data for single crystal manganites having different composition; the excellent agreement demonstrates the validity of the model; points are experimental data taken from literature [57].

[32] and are consistent with the picture developed by Millis et al. [69] for manganites; moreover, when we compute $\gamma$ with the method described in literature [64], we obtain $\gamma=0.32$.

The phonon we have chosen corresponds to a temperature of $600 \mathrm{~K}$, which is about twice the observed MIT temperature, below which the polaron picture is applicable [20,21], and to an energy of $0.05 \mathrm{eV}$, which is close to the one used in a recent theory of colossal magnetoresistance (CMR) manganites [31].

Figure 3 shows the transport data and fits for a 40-nm-thick LSMO thin film deposited on NGO. This case is representative for all the samples. It is also clear that the polynomial fit approach (pink line) fails to follow the data above $120 \mathrm{~K}$ and that the $T^{5}$ coefficient is unphysically low, about nine orders of magnitude lower than the $T^{2}$ coefficient (we use the $R_{5}>0$ constraint in the numerical fit).

We would like to emphasize that the term describing the polaron contribution to the resistance differs from the one used by Varshney and Kaurav [35] for the optical phonon by solving the Bloch-Grüneisen integral in the assumption of an Einstein phonons distribution for the $\exp \left[2 g^{2} \operatorname{coth}\left(\frac{\hbar \omega_{0}}{2 k_{B} T}\right)\right]$ term, confirming that the proper consideration of the temperature effect on the polaron bandwidth was the missing element in their work. Moreover, it is possible to observe by few steps that $\frac{\exp \left(\frac{\hbar \omega_{0}}{k_{B} T}\right)}{\left(\exp \left(\frac{\hbar \hbar_{0}}{k_{B} T}\right)-1\right)^{2}}=\frac{1}{4 \sinh ^{2}\left(\frac{\hbar \omega_{0}}{2 k_{B} T}\right)}$, which is the dominant temperature dependence of the scattering time for the small polaron tunnelling in the Lang-Firsov theory [47].

Transport behavior above the MIT is now addressed. It is mandatory to specify that the existence of a MIT by itself is still a question of debate: single crystals sometimes seem to feature a metal-to-metal transition, and the phase above $T_{C}$ was identified as a paramagnetic metal [70], but the samples were measured up to only $500 \mathrm{~K}$, while measurements up to $1100 \mathrm{~K}$ featured an extremely broad MIT [28]. We propose to define a metal-to-metal transition only when the criterion $\frac{d^{2} \rho}{d T^{2}}>0$ is satisfied above the transition and not when it simply is $\frac{d \rho}{d T}>0$, since the condition when $\frac{d \rho}{d T}>0$ but $\frac{d^{2} \rho}{d T^{2}}<0$ can just be the beginning of a (broad) metal-to-insulator transition and the behavior cannot be regarded as metallic, although $\frac{d \rho}{d T}>0$. A metal-metal transition was found only in the $35 \mathrm{~nm}$ LSMO film grown on STO; thus, we are not able to solve this 
TABLE I. Relevant fit parameters for epitaxial films - this work.

\begin{tabular}{lcccccccc}
\hline \hline$t(\mathrm{~nm})$ & Substrate & $R_{0}(\Omega)$ & $R_{2}\left(\Omega / \mathrm{K}^{2}\right)$ & $C_{F M}(\Omega)$ & $\gamma$ & $\Delta, C_{P M}$ or $R_{5}$ & $\Gamma(\mathrm{K})$ \\
\hline 40 & NGO & 32 & $2 \times 10^{-3}$ & $9 \times 10^{-2}$ & 0.27 & $R_{5}=3 \times 10^{-11} \Omega / \mathrm{K}^{5}$ & - \\
5 & STO & 440 & $2.5 \times 10^{-2}$ & 0.19 & 0.33 & $\Delta=0.16 \mathrm{eV}$ & 0.9999 \\
9 & STO & 750 & $4 \times 10^{-2}$ & $6.4 \times 10^{-2}$ & 0.33 & $\Delta=0.10 \mathrm{eV}$ & 35.2 & 1 \\
13 & STO & 155 & $8 \times 10^{-3}$ & 3.0 & 0.19 & $\Delta=0.12 \mathrm{eV}$ & 28.1 & 0.9995 \\
20 & STO & 110 & $8 \times 10^{-3}$ & $5.5 \times 10^{-3}$ & 0.35 & $\Delta=0.14 \mathrm{eV}$ & 46.2 & 0.9989 \\
35 & STO & 30 & $2 \times 10^{-2}$ & $2.8 \times 10^{-4}$ & 0.35 & $C_{P M}=10^{-3} \Omega$ & 15.5 & 0.9999 \\
\hline \hline
\end{tabular}

debate, but we will show that the model we propose works also in this scenario.

Concerning the case of a MIT, the data above the MIT can be linearized by plotting $\ln (R / T)$ versus $1 / T$ (inset to Fig. 3), indicating standard adiabatic hopping in agreement with both experimental data [28] and polaron theory [20]. In particular, this is consistent with the bipolaron theory developed for CMR and enables us to use the CCDC picture to describe the MIT [31,33,71]. The equation for the insulating branch of $R(T)$ is therefore $R_{I}(T)=A T \exp \left(-\frac{\Delta}{2 k_{B} T}\right)$, where $A$ is a constant and $\Delta$ is the bipolaron binding energy. We find $\Delta \sim 0.1 \mathrm{eV}$, consistent with the known values for manganites [24,33] and confirming, when compared with LSMO samples having different grain sizes distributions [72], the general high degree of structural order of the samples.

The overall $R(T)$ is described by

$$
R(T)=R_{M}^{f(T)}(T) \cdot R_{I}^{1-f(T)}(T),
$$

where $R_{M}$ and $R_{I}$ are the fitting equations below and above the MIT and $f(T)=\left\{\frac{1}{2}\left(1-\operatorname{erf}\left(\frac{T-T_{t}}{\Gamma}\right)\right)\right\}^{\varsigma}$ represents the fraction of metallic phase; note that a nonideality exponent $\varsigma$ is added in respect to the original CCDC model $[31,33]$. This way, to mix $R_{M}$ and $R_{I}$ takes count of a phase separation scenario [73], which is known to happen during the MIT in LSMO [74]. The whole $R(T)$ is fitted by setting the parameters obtained for the two separate $R_{M}(T)$ and $R_{I}(T)$ branches as constants so that in this final step, the only fitting parameters are those in $f$.

Concerning the case of a metal-metal transition, we first have to stress that a theory that consistently explains such a transition does not exist, and all the theories for manganites predict a transition towards an insulating state $[10,31,33,69,75,76]$. Nevertheless, the data above the transition, corresponding to a supposed paramagnetic metallic phase, [70] are described with the electron-phonon coupling term but considering a constant effective mass (since the polaron band picture with the exponential increase of the effective mass is broken) and the temperature acting only on the phonon population $n_{\omega}$. This leads to $R_{P M}(T)=R_{0, P M}+$ $C_{P M} n_{\omega}\left(n_{\omega}+1\right)$, where $P M$ stands for paramagnetic metal phase [70]. In Eq. (3) $R_{I}$ will be substituted by $R_{P M}$.

Figures 4 and 5 show the fits of the insulating phase (red line) for a few films of various thicknesses $t$ deposited on STO and the most relevant fit parameters are reported in Table I. The high value of the $\chi_{s}$, close, or even equal, to 1 , is due not only to the goodness of the model but also to the high number of data points (some ten thousands), which permits us to consider some possible noise, as in the 20-nm-thick sample, simply as an enlarged error bar. For the samples featuring the
MIT, $\Delta$ is shown, while for the sample featuring a metal-metal transition, the $C_{P M}$ coefficient is reported and for the $40 \mathrm{~nm}$ film on NGO the values of the (ruled out) $R_{5}$ coefficient is also reported. The transition width $\Gamma$ is relatively broad $(25-40 \mathrm{~K})$, in agreement with $M(T)$ measurements [48].

The low value of the nonideality exponent $(\varsigma \approx 1.03)$ confirms the suitability of the CCDC model. In the CCDC picture, the carrier density is almost constant up to a temperature of $0.9 \cdot T_{c}$ in LSMO [37], showing that the constant carrier density approximation is realistic in the metallic side; it collapses just when the polaron band picture breaks down.

Figure 5 shows also the fit for the higher temperature region in the case of a metal-to-metal transition. We obtain a transition temperature of $333 \mathrm{~K}$, which gives sense to the $C_{P M} / C_{F M}$ ratio value. In fact, the polaron effective mass increase term $\exp \left[2 g^{2} \operatorname{coth}\left(\frac{\hbar \omega_{0}}{2 k_{B} T}\right)\right]$ at the transition temperature is $1.05 \times 10^{6}$, which is of the same order of the $C_{P M} / C_{F M}$ ratio, meaning that once the effective mass increase breaks the polaron band picture, the carriers conserve their effective mass.

In order to demonstrate the validity and the general relevance of the proposed polaron metal transport model, the model is applied to the metallic phase of single crystals of LCMO, LCPMO, and LSMO, taking the data from the literature [57], and the results are shown in Fig. 6. The fits have been conducted as for LSMO films: data are linearized by plotting $\rho$ versus $T^{2}$ and with the obtained coefficients, Eq. (2) is applied from the lowest temperature value to $0.9 \cdot T_{M I}$. The parameters of the numerical fit are $C_{P M}$ and $g^{2}$, and we impose the phonon $\hbar \omega_{0}$. While the characteristic mode for rhombohedral manganite is $\sim 420 \mathrm{~cm}^{-1}$, for orthorhombic manganites, it is $\sim 230 \mathrm{~cm}^{-1}$ [11], so for LCMO, the dominant mode $\hbar \omega_{0}=235 \mathrm{~cm}^{-1}$ [11] has been chosen. For LCPMO, although the spectroscopic data are absent, we extrapolate $\hbar \omega_{0}=243 \mathrm{~cm}^{-1}$, considering its $T_{C}$ [70] and the trend for orthorhombic manganites [11,57]; for LSMO $\hbar \omega_{0}=425 \mathrm{~cm}^{-1}$ has been kept. It is possible to observe the excellent agreement for both LPCM and LSMO_-so, concerning LSMO, the model is extended also to single crystals grown and measured by other groups. Moreover the slight discrepancy in fitting the LCMO data near the MIT is solved when the data on LCMO in the whole temperature range are fitted using the CCDC model. Fit parameters are reported in Table II. It is possible to observe that the fit accuracy is poorer and $\gamma$ is lower than the one for the film deposited in this work. These facts can be ascribed to both the single crystal nature of the samples (intrinsic nature of the materials) or to some imprecision in the digitalization of the original published figure (extrinsic causes). 
TABLE II. Relevant fit parameters for single crystals—experimental data from Ref. [57].

\begin{tabular}{lcccccc}
\hline \hline Compound & $\rho_{0}(\Omega \mathrm{cm})$ & $\rho_{2}\left(\Omega \mathrm{cm} / \mathrm{K}^{2}\right)$ & $C_{F M}(\Omega \mathrm{cm})$ & $\gamma$ & $\Delta(\mathrm{eV})$ & $\Gamma(\mathrm{K})$ \\
\hline LCMO & $1.4 \times 10^{-4}$ & $1.4 \times 10^{-8}$ & $1 \times 10^{-9}$ & 0.18 & 0.16 & 5.5 \\
LCPMO & $9.5 \times 10^{-5}$ & $1.0 \times 10^{-8}$ & $2.5 \times 10^{-10}$ & 0.16 & - & 0.996 \\
LSMO & $5.4 \times 10^{-5}$ & $7.4 \times 10^{-9}$ & $7.7 \times 10^{-7}$ & 0.18 & - & - \\
\hline \hline
\end{tabular}

In summary, we demonstrated that by taking accurately into account the polaron concepts, a comprehensive description and an excellent fit of charge transport characteristics in manganite thin films is achieved both below and above the MIT. This finding by itself cannot represent sufficient proof for strong polaron effects in LSMO. Nevertheless, it adds significant value to optical-based proofs promoting polarons as dominant carriers in the ferromagnetic and metallic phase of LSMO, other similar manganites, and, feasibly, in other strongly correlated oxide compounds featuring the MIT.
The authors P.G., A.G., M.P., A.R., and I.B. thank F. Bona for technical help and A. Dediu and V. Kabanov for fruitful discussions. Financial support from the FP7 Projects No. NMP3LA-2010-246102 (Interfacing oxides, IFOX), No. NMP-2010SMALL-4-263104 (Next generation hybrid interfaces for spintronic applications, HINTS), No. NMP3-SL-2010-246073 (Graphene for nanoscaled applications, GRENADA), and the Italian government FIRB (Molecular nanomagnets on metallic and magnetic surfaces for applications in molecular spintronics) Project No. RBAP117RWN is acknowledged.
[1] M. Prezioso, A. Riminucci, P. Graziosi et al., Adv. Mater. 25, 534 (2013).

[2] A. Riminucci, M. Prezioso, C. Pernechele, P. Graziosi, I. Bergenti, R. Cecchini, M. Calbucci, M. Solzi, and V. A. Dediu, Appl. Phys. Lett. 102, 092407 (2013).

[3] V. A. Dediu, L. E. Hueso, I. Bergenti, and C. Taliani, Nat. Mater. 8, 707 (2009).

[4] C. Zener, Phys. Rev. 82, 403 (1951).

[5] A. Chattopadhyay, A. J. Millis, and S. Das Sarma, Phys. Rev. B 61, 10738 (2000).

[6] A. J. Millis, P. B. Littlewood, and B. I. Shraiman, Phys. Rev. Lett. 74, 5144 (1995).

[7] A. S. Alexandrov, G. M. Zhao, H. Keller, B. Lorenz, Y. S. Wang, and C. W. Chu, Phys. Rev. B 64, 140404(R) (2001).

[8] C. Hartinger, F. Mayr, J. Deisenhofer, A. Loidl, and T. Kopp, Phys. Rev. B 69, 100403(R) (2004).

[9] C. Hartinger, F. Mayr, A. Loidl, and T. Kopp, Phys. Rev. B 73, 024408 (2006).

[10] A. J. Millis, B. I. Shraiman, and R. Mueller, Phys. Rev. Lett. 77, 175 (1996).

[11] F. Rivadulla, M. Otero-Leal, A. Espinosa, A. de Andrés, C. Ramos, J. Rivas, and J. B. Goodenough, Phys. Rev. Lett. 96, 016402 (2006).

[12] J.-S. Zhou, J. B. Goodenough, A. Asamitsu, and Y. Tokura, Phys. Rev. Lett. 79, 3234 (1997).

[13] L. Sudheendra, V. Moshnyaga, E. D. Mishina, B. Damaschke, T. Rasing, and K. Samwer, Phys. Rev. B 75, 172407 (2007).

[14] F. Massee, S. de Jong, Y. Huang, W. K. Siu, I. Santoso, A. Mans, A. T. Boothroyd, D. Prabhakaran, R. Follath, A. Varykhalov, L. Patthey, M. Shi, J. B. Goedkoop, and M. S. Golden, Nat. Phys. 7, 978 (2011).

[15] F. Weber, N. Aliouane, H. Zheng, J. F. Mitchell, D. N. Argyriou, and D. Reznik, Nat. Mater. 8, 798 (2009).

[16] H. Boschker, M. Huijben, A. Vailionis, J. Verbeeck, S. van Aert, M. Luysberg, S. Bals, G. van Tendeloo, E. P. Houwman, G. Koster, D. H. A. Blank, and G. Rijnders, J. Phys. D 44, 205001 (2011).

[17] I. Mansuri and D. Varshney, J. Alloys Compd. 513, 256 (2012).
[18] M. W. Shaikh and D. Varshney, Mater. Chem. Phys. 134, 886 (2012).

[19] I. G. Austin and N. F. Mott, Adv. Phys. 50, 757 (2001).

[20] J. Appel, in Solid State Physics, edited by D. T. Frederick Seitz and E. Henry (Academic Press, New York, 1968), Vol. 21, p. 193.

[21] G. L. Sewell, Philos. Mag. 3, 1361 (1958).

[22] J. M. Ziman, Electrons and Phonons: The Theory of Transport Phenomena in Solids (Clarendon Press, Oxford, 1960).

[23] A. Chikamatsu, H. Wadati, H. Kumigashira, M. Oshima, A. Fujimori, N. Hamada, T. Ohnishi, M. Lippmaa, K. Ono, M. Kawasaki, and H. Koinuma, Phys. Rev. B 73, 195105 (2006).

[24] M. Egilmez, K. H. Chow, and J. Jung, Appl. Phys. Lett. 92, 162515 (2008).

[25] M. Jaime, P. Lin, S. H. Chun, M. B. Salamon, P. Dorsey, and M. Rubinstein, Phys. Rev. B 60, 1028 (1999).

[26] Y. K. Lakshmi, K. Raju, and P. V. Reddy, J. Appl. Phys. 113, 163701 (2013).

[27] N. Panwar, D. K. Pandya, A. Rao, K. K. Wu, N. Kaurav, Y.-K. Kuo, and S. K. Agarwal, Eur. Phys. J. B 65, 179 (2008).

[28] G. J. Snyder, R. Hiskes, S. DiCarolis, M. R. Beasley, and T. H. Geballe, Phys. Rev. B 53, 14434 (1996).

[29] P. D. Desai, T. K. Chu, H. M. James, and C. Y. Ho, J. Phys. Chem. Ref. Data 13, 1069 (1984).

[30] J. R. Sambles, K. C. Elsom, and G. Sharp-Dent, J. Phys. F 11, 1075 (1981).

[31] A. S. Alexandrov and A. M. Bratkovsky, J. Phys.: Condens. Matter 11, 1989 (1999).

[32] A. S. Alexandrov and A. M. Bratkovsky, Phys. Rev. Lett. 84, 2043 (2000).

[33] A. S. Alexandrov, A. M. Bratkovsky, and V. V. Kabanov, Phys. Rev. Lett. 96, 117003 (2006).

[34] D. Varshney and N. Dodiya, Mater. Chem. Phys. 129, 896 (2011).

[35] D. Varshney and N. Kaurav, Eur. Phys. J. B 40, 129 (2004).

[36] K. Kubo and N. Ohata, J. Phys. Soc. Jpn. 33, 21 (1972).

[37] W. Westerburg, F. Martin, P. J. M. van Bentum, J. A. A. J. Perenboom, and G. Jakob, Eur. Phys. J. B 14, 509 (2000). 
[38] S. Mercone, C. A. Perroni, V. Cataudella et al., Phys. Rev. B 71, 064415 (2005).

[39] D. Varshney, D. Choudhary, M. W. Shaikh, and E. Khan, Eur. Phys. J. B 76, 327 (2010).

[40] D. Varshney and N. Dodiya, Solid State Sci. 13, 1623 (2011).

[41] D. Varshney, N. Dodiya, and M. W. Shaikh, J. Alloys Compd. 509, 7447 (2011).

[42] D. Varshney, M. W. Shaikh, and I. Mansuri, J. Alloys Compd. 486, 726 (2009).

[43] D. Varshney, D. Choudhary, and M. W. Shaikh, Comput. Mater. Sci. 47, 839 (2010).

[44] G. M. Zhao, D. J. Kang, W. Prellier, M. Rajeswari, H. Keller, T. Venkatesan, and R. L. Greene, Phys. Rev. B 63, 060402(R) (2000).

[45] G. M. Zhao, H. Keller, and W. Prellier, J. Phys.: Condens. Matter 12, L361 (2000).

[46] I. G. Lang and Yu. A. Firsov, Zh. Eksp. Teor. Fiz. 43, 1843 (1962) [Sov. Phys.-JETP 16, 1301 (1963)].

[47] I. G. Lang and Yu. A. Firsov, Sov. Phys. JETP 16, 1301 (1963); Sov. Phys. Solid State 5, 2049 (1964).

[48] P. Graziosi, M. Prezioso, A. Gambardella, C. Kitts, R. K. Rakshit, A. Riminucci, I. Bergenti, F. Borgatti, C. Pernechele, M. Solzi, D. Pullini, D. Busquets-Mataix, and V. A. Dediu, Thin Solid Films 534, 83 (2013).

[49] Keithley, 386 User Manual, http://www.keithley.com/ knowledgecenter/knowledgecenter_pdf/LowLevMsHandbk.pdf

[50] F. Borgatti, F. Offi, P. Torelli, G. Monaco, and G. Panaccione, J. Electron Spectrosc. Relat. Phenom. 190, 228 (2013).

[51] P. Orgiani, C. Adamo, C. Barone, A. Galdi, A. Y. Petrov, D. G. Schlom, and L. Maritato, Phys. Rev. B 76, 012404 (2007).

[52] P. Orgiani, A. Yu. Petrov, R. Ciancio, A. Galdi, L. Maritato, and B. A. Davidson, Appl. Phys. Lett. 100, 042404 (2012).

[53] P. Postorino, A. Congeduti, P. Dore, A. Sacchetti, F. Gorelli, L. Ulivi, A. Kumar, and D. D. Sarma, Phys. Rev. Lett. 91, 175501 (2003).

[54] R. Di Capua, C. A. Perroni, V. Cataudella, F. Miletto Granozio, P. Perna, M. Salluzzo, U. Scotti di Uccio, and R. Vaglio, J. Phys.: Condens. Matter 18, 8195 (2006).

[55] A. M. Haghiri-Gosnet and J. P. Renard, J. Phys. D 36, R127 (2003).
[56] L. J. van der Pauw, Philips Tech. Rev. 20, 220 (1958).

[57] Y. Lyanda-Geller, S. H. Chun, M. B. Salamon, P. M. Goldbart, P. D. Han, Y. Tomioka, A. Asamitsu, and Y. Tokura, Phys. Rev. B 63, 184426 (2001).

[58] N. W. Ashcroft and N. D. Mermin, Solid State Physics (Cornell University Press, Ithaca, NY, 1976).

[59] B. Brandow, Phys. Rep. 296, 1 (1998).

[60] M. Gurvitch, A. K. Ghosh, H. Lutz, and M. Strongin, Phys. Rev. B 22, 128 (1980).

[61] P. C. Klipstein, A. G. Bagnall, W. Y. Liang, E. A. Marseglia, and R. H. Friend, J. Phys. C: Solid State Phys. 14, 4067 (1981).

[62] A. H. Thompson, Phys. Rev. Lett. 35, 1786 (1975).

[63] D. van der Marel, J. L. M. van Mechelen, and I. I. Mazin, Phys. Rev. B 84, 205111 (2011).

[64] A. S. Alexandrov, Phys. Rev. B 53, 2863 (1996).

[65] J. T. Devreese and A. S. Alexandrov, Rep. Prog. Phys. 72, 066501 (2009).

[66] E. Arisi, I. Bergenti, V. Dediu, and G. Ruani, J. Magn. Magn. Mater. 272-276, 1751 (2004).

[67] A. Dubroka, J. Humlíček, M. V. Abrashev, Z. V. Popović, F. Sapiña, and A. Cantarero, Phys. Rev. B 73, 224401 (2006).

[68] A. S. Alexandrov, Phys. Rev. B 61, 12315 (2000).

[69] A. J. Millis, R. Mueller, and B. I. Shraiman, Phys. Rev. B 54, 5405 (1996).

[70] A. Urushibara, Y. Moritomo, T. Arima, A. Asamitsu, G. Kido, and Y. Tokura, Phys. Rev. B 51, 14103 (1995).

[71] A. S. Alexandrov and A. M. Bratkovsky, Phys. Rev. Lett. 82, 141 (1999).

[72] D. Salazar, D. Arias, O. J. Durá, and M. A. López de la Torre, J. Alloys Compd. 583, 141 (2014).

[73] V. V. Kabanov, K. Zagar, and D. Mihailovic, J. Exp. Theor. Phys. 100, 715 (2005).

[74] T. Becker, C. Streng, Y. Luo, V. Moshnyaga, B. Damaschke, N. Shannon, and K. Samwer, Phys. Rev. Lett. 89, 237203 (2002).

[75] T. V. Ramakrishnan, H. R. Krishnamurthy, S. R. Hassan, and G. Venketeswara Pai, Phys. Rev. Lett. 92, 157203 (2004).

[76] P. Sanyal, V. B. Shenoy, H. R. Krishnamurthy, and T. V. Ramakrishnan, Eur. Phys. J. B 81, 393 (2011). 